Pacific Journal of Mathematics

SOME PROPERTIES OF THE CHARACTERISTIC OF 


\title{
SOME PROPERTIES OF THE CHARACTERISTIC OF CONVEXITY RELATING TO FIXED POINT THEORY
}

\author{
DAVID J. DOWNING AND BARRY TURETT
}

\begin{abstract}
Fixed point theorems for uniformly lipschitzian mappings often restrict the characteristic of convexity, $\varepsilon_{0}(X)$, of the underlying Banach space to be less than one. This condition is discussed; in particular, it is shown that, for Banach spaces, $\varepsilon_{0}(x)<1$ is equivalent to a condition imposed by $\mathbf{E}$. A. Lifschitz in arbitrary metric spaces. The stability of this condition with respect to Banach-Mazur distance and Lebesgue-Bochner function spaces is also considered.
\end{abstract}

Let $K$ be a nonempty, closed, bounded, convex subset of a Banach space $X$. A mapping $T: K \rightarrow K$ is said to be uniformly $k$-lipschitzian $(k \geq 1)$ if, for each $x, y$ in $K$ and each natural number $n,\left\|T^{n} x-T^{n} y\right\| \leq$ $k\|x-y\|$. Such mappings provide an intermediate class between the class of nonexpansive mappings and the class of lipschitzian mappings with Lipschitz constant greater than one. It is well-known (cf. [9]) that mappings in this latter class may fail to have fixed points even if the underlying space is Hilbert space and the Lipschitz constant is arbitrarily near one. However, fixed point theorems for uniformly lipschitzian mappings have been obtained by Goebel and Kirk [7], Goebel, Kirk, and Thele [8], and Lifschitz [11]. (See also [6].) In obtaining their results, two formally different geometric conditions are imposed on the space in question. In this paper, the relationship between the two geometric conditions is explored. It is shown that, in Banach spaces, the conditions are qualitatively, although not quantitatively, equivalent. In addition, the stability of these conditions is discussed; in particular, we show that these conditions lift from a Banach space $X$ to the corresponding LebesgueBochner function space $L^{p}(\mu, X)$ for $1<p<\infty$ and $\mu$ an arbitrary measure.

Uniformly lipschitzian mappings were originally considered by Goebel and Kirk [7] and then by Goebel, Kirk, and Thele [8] in a more general semigroup setting. They discovered a relationship between the modulus of convexity of $X$ and fixed points for uniformly lipschitzian mappings. Recall, for a normed linear space $X$, the modulus of convexity of $X$ is the function $\delta_{X}:[0,2] \rightarrow[0,1]$ defined by

$$
\delta_{X}(\varepsilon)=\inf \left\{1-\frac{\|x+y\|}{2}:\|x\| \leq 1,\|y\| \leq 1,\|x-y\| \geq \varepsilon\right\} .
$$


The characteristic of convexity of $X, \varepsilon_{0}(X)$, is then defined to be $\sup \left\{\varepsilon \in[0,2]: \delta_{X}(\varepsilon)=0\right\}$. It is well-known that $X$ is uniformly rotund (respectively, uniformly non-square) if and only if $\varepsilon_{0}(X)=0$ [4, p. 145] (respectively, $\varepsilon_{0}(X)<2[4$, p. 146]). The main idea of [7] and [8] may be stated as follows:

Theorem 1 (Goebel, Kirk, Thele). Let $X$ be a Banach space with $\varepsilon_{0}(X)<1$ and let $\gamma>1$ satisfy $\gamma\left(1-\delta_{X}(1 / \gamma)\right)=1$. If $K$ is a nonempty, closed, bounded, convex subset of $X$ and $T: K \rightarrow K$ is uniformly $k$-lipschitzian for $k<\gamma$, then $T$ has a fixed point in $K$.

In a subsequent development, Lifschitz [11] initiated a more topological approach and considered uniformly lipschitzian mappings in metric spaces. Instead of using the modulus of convexity, Lifschitz associated, with each metric space $(M, \rho)$, a constant $\kappa(M)$ defined as follows:

$$
\begin{aligned}
& \kappa(M)=\sup \{\beta>0: \exists \alpha>1 \text { such that } \forall x, y \in M \text { and } r>0, \\
& \rho(x, y)>r \Rightarrow \exists z \in M \text { such that } B(x, \beta r) \cap B(y, \alpha r) \subset B(z, r)\},
\end{aligned}
$$

where $B(x, r)$ denotes the closed ball of radius $r$ centered at $x$. It is immediate that $\kappa(M) \geq 1$ for any metric space $(M, \rho)$. Lifschitz proved that if $(M, \rho)$ is a bounded, complete metric space and if $T: M \rightarrow M$ is uniformly $k$-lipschitzian for $k<\kappa(M)$, then $T$ has a fixed point in $M$. In order to compare this to the Goebel-Kirk-Thele resulting in the setting of Banach spaces, define $\kappa_{0}(X)$ to be the infimum of $\kappa(C)$ where $C$ ranges over all nonempty, closed, bounded, convex subsets of the Banach space $X$. Then Lifschitz's theorem implies:

THeOREM 2 (Lifschitz). Let $X$ be a Banach space with $\kappa_{0}(X)>1$. If $K$ is a nonempty, closed, bounded, convex subset of $X$ and $T: K \rightarrow K$ is uniformly $k$-lipschitzian for $k<\kappa_{0}(X)$, then $T$ has a fixed point in $K$.

Lifschitz proved that $\kappa_{0}(\mathcal{H}) \geq \sqrt{2}$ where $\mathcal{H}$ denotes Hilbert space; Goebel, Kirk, and Thele noted that $\gamma=\sqrt{5} / 2$ is the solution to $\gamma\left(1-\delta_{\mathcal{H}}(1-\gamma)\right)=1$. Thus, for Hilbert spaces, Lifschitz's approach yields a sharper result on how large the Lipschitz constant $k$ may be taken and still guarantee the mappings have fixed points. It should be mentioned that J. Baillion (cf. [8]) has found an example of a fixed point-free uniformly $\pi / 2$-lipschitzian self-mapping defined on a closed, bounded, convex subset of $l^{2}$. Our first result states that the approach of Lifschitz will always provide estimates on the size of $k$ at least as good as those found using the approach of Goebel-Kirk-Thele. 
THEOREM 3. Let $X$ be a Banach space and assume $\gamma>1$ satisfies $\gamma\left(1-\delta_{X}(1 / \gamma)\right)=1$. Then $\gamma \leq \kappa_{0}(X)$.

In order to facilitate the proof of Theorem 3, we state a lemma from [11].

LEMMA 4 (Lifschitz). Let $X$ be a normed linear space, Then $\kappa_{0}(X) \geq$ $\sup \{\beta>0:$ for some $\alpha>1$ and all $y \in X$ with $\|y\|>1$, there exists $t \in[0,1]$ with $B(0, \beta) \cap B(y, \alpha) \subset B(t y, 1)\}$.

Proof of Theorem 3. Let $y \in X$ with $\|y\|>1$ and suppose $x \in B(0, \gamma)$ $\cap B(y, \gamma)$. Then $\|x / \gamma\| \leq 1,\|(x-y) / \gamma\| \leq 1$ and $\|x / \gamma-(x-y) / \gamma\|$ $>1 / \gamma$. Therefore, by the definition of $\delta_{X},\|1 / 2(x / \gamma+(x-y) / \gamma)\| \leq$ $1-\delta_{X}(1 / \gamma)$. Thus, $\|x-y / 2\| \leq \gamma\left(1-\delta_{X}(1 / \gamma)\right)=1$; i.e., $x \in$ $B(y / 2,1)$. By Lemma $3, \kappa_{0}(X) \geq \gamma$. This completes the proof of Theorem 4.

Although, in a quantitative sense, Lifschitz's result yields sharper estimates on the size of $k$ than does the Goebel-Kirk-Thele result, the next theorem shows that, in the setting of Banach spaces, the results are qualitatively equivalent.

TheOREM 5. Let $X$ be a Banach space. Then $\varepsilon_{0}(X)<1$ if and only if $\kappa_{0}(X)>1$.

Proof. If $\varepsilon_{0}(X)<1$, it is immediate that $\gamma$ satisfying $\gamma\left(1-\delta_{X}(1 / \gamma)\right)$ $=1$ is greater than 1 . So, by Theorem $4, \kappa_{0}(X) \geq \gamma>1$.

Now assume $\varepsilon_{0}(X) \geq 1$ and let $\beta>1$ and $\alpha>1$. Then there exist norm one elements $x, y$ in $X$ such that $\|x-y\|>1 / \gamma$ and $\|(x+y) / 2\|$ $>1 / \gamma$ where $\gamma=\min \{\alpha, \beta, 2\}>1$. Consider $B(0, \beta) \cap B(\gamma(x-y), \alpha)$. Since $\|\gamma x\|=\gamma \leq \beta$ and $\|\gamma x-\gamma(x-y)\|=\gamma\|y\|=\gamma \leq \alpha, \quad \gamma x \in$ $B(0, \beta) \cap B(\gamma(x-y), \alpha)$. Similarly $-\gamma y \in B(0, \beta) \cap B(\gamma(x-y), \alpha)$. But $\|\gamma x-(-\gamma y)\|=\gamma\|x+y\|>2$ and hence there does not exist $z$ in $X$ with $B(0, \beta) \cap B(\gamma(x-y), \alpha) \subset B(z, 1)$. Since 0 and $\gamma(x-y)$ are in $4 B_{X}$, and $\beta>1$ and $\alpha>1$ are arbitrary, $\kappa\left(4 B_{X}\right)=1$; thus $\kappa_{0}(X)=1$. This completes the proof of Theorem 5 .

The conditions compared in Theorem 5 are, in some senses, stable. Recall that, for isomorphic Banach spaces $X$ and $Y$, the Banach-Mazur distance coefficient from $X$ to $Y$, denoted $d(X, Y)$, is defined to be the infimum of $\|U\|\left\|U^{-1}\right\|$, the infimum being taken over all invertible operators $U$ from $X$ onto $Y$. 
THEOREM 6. Let $X$ be a Banach space with $\varepsilon_{0}(X)<1$ and let $\gamma>1$ satisfy $\gamma\left(1-\delta_{X}(1 / \gamma)\right)=1$. If $Y$ is a Banach space isomorphic to $X$ and $d(X, Y)<\gamma$, then $\varepsilon_{0}(Y)<1$.

Proof. Without loss of generality, let $U$ be an isomorphism from $X$ onto $Y$ such that $\left\|U^{-1}\right\|=1$ and $d(X, Y) \leq\|U\|<\gamma$. Choose norm-one elements $y_{1}$ and $y_{2}$ in $Y$ such that $\left\|y_{1}-y_{2}\right\| \geq\|U\| / \gamma$ and define $x_{1}=$ $U^{-1}\left(y_{1}\right)$ and $x_{2}=U^{-1}\left(y_{2}\right)$. It is immediate that $\left\|x_{1}\right\| \leq 1,\left\|x_{2}\right\| \leq 1$ and $\left\|x_{1}-x_{2}\right\| \geq 1 / \gamma$. Then, by the definition of $\delta_{X},\left\|\left(x_{1}+x_{2}\right) / 2\right\| \leq$ $1-\delta_{X}(1 / \gamma)$. Therefore

$$
\begin{aligned}
\left\|\left(y_{1}+y_{2}\right) / 2\right\| & \leq\|U\|\left\|\left(x_{1}+x_{2}\right) / 2\right\| \\
& \leq\|U\|\left(1-\delta_{X}(1 / \gamma)\right)<\gamma\left(1-\delta_{X}(1 / \gamma)\right)=1 .
\end{aligned}
$$

This implies that $\delta_{Y}(\|U\| / \gamma) \geq 1-\|U\|\left(1-\delta_{X}(1 / \gamma)\right)>0$. Thus $\varepsilon_{0}(Y)$ $\leq\|U\| / \gamma<1$ and the proof is complete.

Theorem 6 allows us to generalize some recent work of Bynum [2]. He has shown that if $X$ is uniformly rotund, then there exists $\beta>1$ so that if $Y$ is a Banach space with $d(X, Y)<\beta$, closed, bounded, convex subsets of $Y$ have the fixed point property for nonexpansive mappings. The next corollary follows immediately from Theorems 1 and 6 .

Corollary 7. Let $X$ be a Banach space with $\varepsilon_{0}(X)<1$. Then there exist constants $\gamma>1$ and $\beta>1$ such that if $Y$ is a Banach space isomorphic to $X$ and $d(X, Y)<\beta$, then closed, bounded, convex subsets of $Y$ have the fixed point property for uniformly $k$-lipschitzian mappings with $k<\gamma$.

Finally we show that these conditions are stable in a second sense.

THEOREM 8. If $X$ is a Banach space with $\varepsilon_{0}(X)<1, \mu$ an arbitrary measure, and $1<p<\infty$, then $\varepsilon_{0}\left(L^{p}(\mu, X)\right)<1$.

Theorem 8 follows immediately from Theorem 9 below. A discussion of Lebesgue-Bochner function spaces may be found in [1] or [5]. If $\mu$ is counting measure over some set; $L^{p}(\mu, X)$ is the Banach sequence space $l^{p}(X)$. The next theorem demonstrates the relationship between the characteristic of convexity of a Banach space $X$ and the characteristic of convexity of the corresponding Lebesgue-Bochner function space. The proof is closely modelled on Day's proof [3] that $L^{p}(\mu, X)$ is uniformly rotund if and only if $X$ is uniformly rotund and $1<p<\infty$.

THEOREM 9. Let $X$ be a Banach space and $\mu$ a measure. Then $\varepsilon_{0}\left(L^{p}(\mu, X)\right)=\max \left\{\varepsilon_{0}\left(l^{p}\right), \varepsilon_{0}(X)\right\}$. 
Proof. Since both $X$ and $L^{p}(\mu)$ are isometric to subspaces of $L^{p}(\mu, X)$, it is clear that

$$
\varepsilon_{0}\left(L^{p}(\mu, X)\right) \geq \max \left\{\varepsilon_{0}\left(L^{p}(\mu)\right), \varepsilon_{0}(X)\right\}=\max \left\{\varepsilon_{0}\left(l^{p}\right), \varepsilon_{0}(X)\right\} .
$$

It is then immediate that if $p=1, p=\infty$, or $\varepsilon_{0}(X)=2$, the desired equality is obtained with $\varepsilon_{0}\left(L^{p}(\mu, X)\right)=2$. Thus, for the remainder of the proof, assume $1<p<\infty$ and $\varepsilon_{0}(X)<2$. Since $\varepsilon_{0}\left(l^{p}\right)=0$ for $1<p$ $<\infty$, it suffices to demonstrate that $\varepsilon_{0}\left(L^{p}(\mu, X)\right) \leq \varepsilon_{0}(X)$.

Assume further that $\mu$ is counting measure on the set of natural numbers. Although this assumption appears quite restrictive, once the theorem is verified for counting measure, the theorem follows quickly for an arbitrary measure $\mu$ by defining an embedding of simple functions in $L^{p}(\mu, X)$ into the space $l^{p}(X)$ as done by Day [3, p. 507]. Thus it suffices to prove that $\varepsilon_{0}\left(l^{p}(X)\right) \leq \varepsilon_{0}(X)$ for $1<p<\infty$ and $\varepsilon_{0}(X)<2$.

Let $b=\left(x_{i}\right)$ and $b^{\prime}=\left(x_{i}^{\prime}\right)$ be elements of $l^{p}(X)$ and let $0<\eta \leq 2$. First, consider the case $\|b\|=\left\|b^{\prime}\right\|=1,\left\|b-b^{\prime}\right\| \geq \varepsilon_{0}(X)+\mu$, and $\left\|x_{i}\right\|$ $=\left\|x_{i}^{\prime}\right\|$ for all $i \in \mathbf{N}$. For convenience, let $\left\|x_{i}\right\|=\beta_{i}$ and $\left\|x_{i}-x_{i}^{\prime}\right\|=\gamma_{i}$. Then

$$
\left\|b+b^{\prime}\right\|=\left(\sum_{i}\left\|x_{i}+x_{i}^{\prime}\right\|^{p}\right)^{1 / p} \leq 2\left\{\sum_{i}\left[\left(1-\delta_{X}\left(\frac{\gamma_{i}}{\beta_{i}}\right)\right) \beta_{i}\right]^{p}\right\}^{1 / p} .
$$

Note that $\gamma_{i} \leq 2 \beta_{i}$ for each $i \in \mathbf{N}$. Define $E=\left\{i \in \mathbf{N}: \gamma_{i} / \beta_{i}>\right.$ $\left.2\left(\varepsilon_{0}(X)+\eta\right) /(2+\eta)\right\}$ and $F=\mathbf{N} \backslash E$. Then

$$
1=\left(\sum_{i} \beta_{i}^{p}\right)^{1 / p} \geq\left(\sum_{i \in F} \beta_{i}^{p}\right)^{1 / p} \geq \frac{2+\eta}{2\left(\varepsilon_{0}(X)+\eta\right)}\left(\sum_{i \in F} \gamma_{i}^{p}\right)^{1 / p}
$$

that is,

$$
\left(\sum_{i \in F} \gamma_{i}^{p}\right)^{1 / p} \leq \frac{2\left(\varepsilon_{0}(X)+\eta\right)}{2+\eta}
$$

or

$$
\left(\sum_{i \in E} \gamma_{i}^{p}\right)^{1 / p}=\left(\sum_{i} \gamma_{i}^{p}-\sum_{i \in F} \gamma_{i}^{p}\right)^{1 / p} \geq\left(\varepsilon_{0}(X)+\eta\right)\left(1-\left(\frac{2}{2+\eta}\right)^{p}\right)^{1 / p} .
$$

Hence

$$
\alpha=\left(\sum_{i \in E} \beta_{i}^{p}\right)^{1 / p} \geq \frac{1}{2}\left(\sum_{i \in E} \gamma_{i}^{p}\right)^{1 / p} \geq \frac{\eta}{2}\left(1-\left(\frac{2}{2+\eta}\right)^{p}\right)^{1 / p}
$$


Thus,

$$
\begin{aligned}
& \left\|b+b^{\prime}\right\| \leq 2\left\{\left(1-\delta_{X}\left(\frac{2\left(\varepsilon_{0}(X)+\eta\right)}{2+\eta}\right)\right)^{p} \sum_{i \in E} \beta_{i}^{p}+\sum_{i \in F} \beta_{i}^{p}\right\}^{1 / p} \\
& =2\left\{\left(1-\delta_{X}\left(\frac{2\left(\varepsilon_{0}(X)+\eta\right)}{2+\eta}\right)\right)^{p} \alpha^{p}+1-\alpha^{p}\right\}^{1 / p} \\
& =2\left\{1-\left[1-\left(1-\delta_{X}\left(\frac{2\left(\varepsilon_{0}(X)+\eta\right)}{2+\eta}\right)\right)^{p}\right] \alpha^{p}\right\}^{1 / p} \\
& \leq 2\left\{1-\left[1-\left(1-\delta_{X}\left(\frac{2\left(\varepsilon_{0}(X)+\eta\right)}{2+\eta}\right)\right)^{p}\right]\left(\frac{\eta}{2}\right)^{p}\left(1-\left(\frac{2}{2+\eta}\right)^{p}\right)\right\}^{1 / p} .
\end{aligned}
$$

Since $0<\eta \leq 2$ and $2\left(\varepsilon_{0}(X)+\eta\right) /(2+\eta)>\varepsilon_{0}(X)$, if we set the righthand side equal to $2\left(1-\delta_{0}\left(\varepsilon_{0}(X)+\eta\right)\right)$, then $\delta_{0}\left(\varepsilon_{0}(X)+\eta\right)>0$ for all $\eta>0$. Thus the first case is finished.

Now suppose $\|b\|=\left\|b^{\prime}\right\|=1$ and assume $\left\|b+b^{\prime}\right\|>2\left(1-\delta_{p}(\xi)\right)$ where $\delta_{p}$ is the modulus of convexity of $l^{p}$ and

$$
\xi=\min \left\{\eta / 4, \frac{1}{2} \delta_{0}\left(\varepsilon_{0}(X)+\eta / 2\right)\right\}>0
$$

Then

$$
2\left(1-\delta_{p}(\xi)\right) \leq\left(\sum_{i}\left\|x_{i}+x_{i}^{\prime}\right\|^{p}\right)^{1 / p} \leq\left(\sum_{i}\left(\left\|x_{i}\right\|+\left\|x_{1}^{\prime}\right\|\right)^{p}\right)^{1 / p} \leq 2 .
$$

Since $\left(\left\|x_{i}\right\|\right)$ and $\left(\left\|x_{i}^{\prime}\right\|\right)$ are norm-one elements in $l^{p}$,

$$
\left(\sum_{i}\left|\left\|x_{i}\right\|-\left\|x_{i}^{\prime}\right\|\right|^{p}\right)^{1 / p}<\xi .
$$

Let $b^{\prime \prime}=\left(x_{i}^{\prime}\right)$ where

$$
x_{i}^{\prime \prime}= \begin{cases}\frac{x_{i}^{\prime}\left\|x_{i}\right\|}{\left\|x_{i}^{\prime}\right\|} & \text { if } x_{i}^{\prime} \neq 0 \\ x_{i} & \text { if } x_{i}^{\prime}=0\end{cases}
$$


Note $\left\|x_{i}\right\|=\left\|x_{i}^{\prime \prime}\right\|$ for all $i \in \mathbf{N}$ and $\left\|b^{\prime}-b^{\prime \prime}\right\|=\left(\Sigma\left|\left\|x_{i}^{\prime}\right\|-\left\|x_{i}\right\|\right|^{p}\right)^{1 / p}$ $<\xi<\eta / 2$. Thus,

$$
\begin{aligned}
\left\|b+b^{\prime \prime}\right\| & \geq\left\|b+b^{\prime}\right\|-\left\|b^{\prime}-b^{\prime \prime}\right\| \geq 2\left(1-\delta_{p}(\xi)\right)-\xi \\
& =2\left(1-\delta_{p}(\xi)-\frac{\xi}{2}\right) \geq 2\left(1-\frac{\xi}{2}-\frac{\xi}{2}\right), \text { since } \delta_{p}(\xi) \leq \frac{\xi}{2} \\
& =2(1-\xi)>2\left(1-\delta_{0}\left(\varepsilon_{0}(X)+\frac{\eta}{2}\right)\right)
\end{aligned}
$$

It now follows from the first case that $\left\|b-b^{\prime \prime}\right\| \leq \varepsilon_{0}(X)+\eta / 2$. Finally,

$$
\left\|b-b^{\prime}\right\| \leq\left\|b-b^{\prime \prime}\right\|+\left\|b^{\prime \prime}-b^{\prime}\right\|<\varepsilon_{0}(X)+\frac{\eta}{2}+\frac{\eta}{2}=\varepsilon_{0}(X)+\eta .
$$

Therefore, if $\|b\|=\left\|b^{\prime}\right\|=1$ and $\left\|b-b^{\prime}\right\| \geq \varepsilon_{0}(X)+\eta$, then $\left\|\left(b+b^{\prime}\right) / 2\right\| \leq 1-\delta_{p}(\xi)$. This implies that $\delta_{l^{p}(X)}\left(\varepsilon_{0}(X)+\eta\right) \geq \delta_{p}(\xi)>$ 0 . Thus $\varepsilon_{0}\left(l^{p}(X)\right) \leq \varepsilon_{0}(X)+\eta$. Since $\eta>0$ was arbitrary, $\varepsilon_{0}\left(l^{p}(X)\right) \leq$ $\varepsilon_{0}(X)$ and the proof of Theorem 9 is complete.

Theorems 1 and 8 combine to yield the following fixed point theorem.

Corollary 10. Let $X$ be a Banach space with $\varepsilon_{0}(X)<1, \mu$ a measure, and $1<p<\infty$. Then there exists a constant $\gamma>1$ so that closed, bounded, convex subsets of the Lebesgue-Bochner function space $L^{p}(\mu, X)$ have the fixed-point property for uniformly $k$-lipschitzian mappings with $k<\gamma$.

Since $X$ is uniformly rotund if and only if $\varepsilon_{0}(X)=0$, Day's result follows immediately from Theorem 9 .

Corollary 11 (Day [3]). Let $(\Omega, \Sigma, \mu)$ be a measure space. The Lebesgue-Bochner function space $L^{p}(\mu, X)$ is uniformly rotund if and only if $1<p<\infty$ and $X$ is uniformly rotund.

In a similar vein, Theorem 9 together with the characterization of uniformly non-square spaces in terms of the characteristic of convexity mentioned prior to Theorem 1 combine to prove the next corollary.

COROLlaRY 12 (Smith-Turett $[12, p .116])$. Let $(\Omega, \Sigma, \mu)$ be a measure space. The Lebesgue-Bochner function space $L^{p}(\mu, X)$ is uniformly nonsquare if and only if $1<p<\infty$ and $X$ is uniformly non-square. 


\section{REFERENCES}

1. S. Bochner and A. E. Taylor, Linear functionals on certain spaces of abstractly-valued functions, Ann. of Math., (2) 39 (1938), 913-944.

2. W. L. Bynum, Normal structure coefficients for Banach spaces, Pacific J. Math., 86 (1980), 427-436.

3. M. M. Day, Some more uniformly convex spaces, Bull. Amer. Math. Soc., 47 (1941), 504-507.

4. __, Normed Linear Spaces, 3rd Ed., Ergebnisse Mathematik und ihrer Grenzgebiete, Band 21, Springer-Verlag, New York, 1973.

5. J. Diestel and J. J. Uhl, Jr., Vector Measures, Math. Surveys, No. 15, Amer. Math. Soc., Providence, R.I., 1977.

6. D. Downing and W. Ray, Uniformly lipschitzian semigroups in Hilbert space, Canad. Math. Bull., (to appear).

7. K. Goebel and W. A. Kirk, A fixed point theorem for transformations whose iterates have uniform Lipschitz constant, Studia Math., 67 (1973), 135-140.

8. K. Goebel, W. A. Kirk and R. L. Thele, Uniformly lipschitzian families of transformations in Banach spaces, Canad. J. Math., 31 (1974), 1245-1256.

9. W. A. Kirk, A fixed point theorem for mappings which do not increase distances, Amer. Math. Monthly, 72 (1965), 1004-1006.

10. Fixed point theory for nonexpansive mappings, Summer Workshop on Fixed

Point Theory, Université de Sherbrooke, June 1980.

11. E. A. Lifschitz, Fixed point theorems for operators in strongly convex spaces, Voronež Gos. Univ. Trudy Mat. Fak., 16 (1975), 23-28. (Russian)

12. M. A. Smith and B. Turett, Rotundity in Lebesgue-Bochner function spaces, Trans. Amer. Math. Soc., 257 (1980), 105-118.

Received April 20, 1981

OAKLAND UNIVERSITY

ROCHESTER, MI 48063 


\title{
PACIFIC JOURNAL OF MATHEMATICS EDITORS
}

\author{
Donald BABBITT (Managing Editor) \\ University of California \\ Los Angeles, CA 90024 \\ Hugo Rossi \\ University of Utah \\ Salt Lake City, UT 84112 \\ C. C. Moore and Arthur Ogus \\ University of California \\ Berkeley, CA 94720
}

\author{
J. Dugundi \\ Department of Mathematics \\ University of Southern California \\ Los Angeles, CA 90089-1113
}

R. FinN and H. SAMELSON

Stanford University

Stanford, CA 94305

\section{ASSOCIATE EDITORS}
R. ARENS
E. F. BECKENBACH
B. H. NeumanN
F. WOLF
K. YoSHIDA (1906-1982)

\section{SUPPORTING INSTITUTIONS}

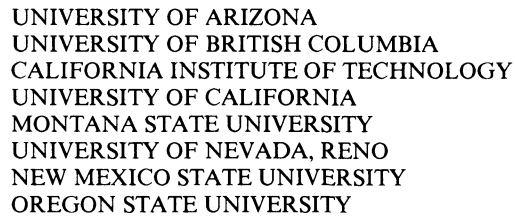

UNIVERSITY OF ARIZONA

UNIVERSITY OF BRITISH COLUMBIA

CALIFORNIA INSTITUTE OF TECHNOLOGY

UNIVERSITY OF CALIFORNIA

MONTANA STATE UNIVERSITY

UNIVERSITY OF NEVADA, RENO

NEW MEXICO STATE UNIVERSITY

OREGON STATE UNIVERSITY

\author{
UNIVERSITY OF OREGON \\ UNIVERSITY OF SOUTHERN CALIFORNIA \\ STANFORD UNIVERSITY \\ UNIVERSITY OF HAWAII \\ UNIVERSITY OF TOKYO \\ UNIVERSITY OF UTAH \\ WASHINGTON STATE UNIVERSITY \\ UNIVERSITY OF WASHINGTON
}

The Supporting Institutions listed above contribute to the cost of publication of this Journal, but they are not owners or publishers and have no responsibility for its content or policies.

Mathematical papers intended for publication in the Pacific Journal of Mathematics should be in typed form or offset-reproduced (not dittoed), double spaced with large margins. Please do not use built up fractions in the text of the manuscript. However, you may use them in the displayed equations. Underline Greek letters in red, German in green, and script in blue. The first paragraph must be capable of being used separately as a synopsis of the entire paper. In particular it should contain no bibliographic references. Please propose a heading for the odd numbered pages of less than 35 characters. Manuscripts, in triplicate, may be sent to any one of the editors. Please classify according to the scheme of Math. Reviews, Index to Vol. 39. Supply name and address of author to whom proofs should be sent. All other communications should be addressed to the managing editor, or Elaine Barth, University of California, Los Angeles, California 90024.

There are page-charges associated with articles appearing in the Pacific Journal of Mathematics. These charges are expected to be paid by the author's University, Government Agency or Company. If the author or authors do not have access to such Institutional support these charges are waived. Single authors will receive 50 free reprints; joint authors will receive a total of 100 free reprints. Additional copies may be obtained at cost in multiples of 50 .

The Pacific Journal of Mathematics is issued monthly as of January 1966. Regular subscription rate: $\$ 132.00$ a year (6 Vol., 12 issues). Special rate: $\$ 66.00$ a year to individual members of supporting institutions.

Subscriptions, orders for numbers issued in the last three calendar years, and changes of address should be sent to Pacific Journal of Mathematics, P.O. Box 969, Carmel Valley, CA 93924, U.S.A. Old back numbers obtainable from Kraus Periodicals Co., Route 100, Millwood, NY 10546.

The Pacific Journal of Mathematics ISSN 0030-8730 is published monthly by the Pacific Journal of Mathematics at P.O. Box 969, Carmel Valley, CA 93924. Application to mail at Second-class postage rates is pending at Carmel Valley, California, and additional mailing offices. Postmaster: Send address changes to Pacific Journal of Mathematics, P. O. Box 969, Carmel Valley, CA 93924.

PUBLISHED BY PACIFIC JOURNAL OF MATHEMATICS, A NON-PROFIT CORPORATION

Copyright $\odot 1983$ by Pacific Journal of Mathematics 


\section{Pacific Journal of Mathematics}

Vol. 104, No. $2 \quad$ June, 1983

Leo James Alex, Simple groups and a Diophantine equation ........... 257

Herbert James Alexander and John Wermer, On the approximation of

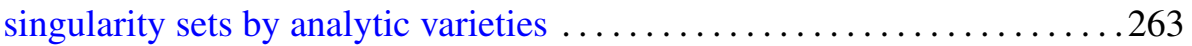

Waleed A. Al-Salam and Mourad Ismail, Orthogonal polynomials associated with the Rogers-Ramanujan continued fraction .......... 269

J. L. Brenner and Roger Conant Lyndon, Permutations and cubic

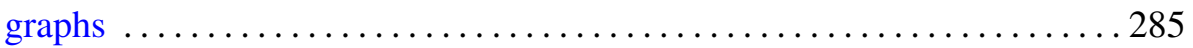

Ian George Craw and Susan Ross, Separable algebras over a commutative

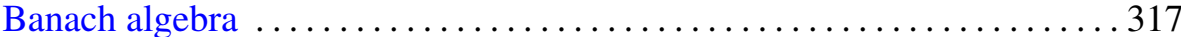

Jesus M. Dominguez, Non-Archimedean Gel'fand theory ............. 337

David Downing and Barry Turett, Some properties of the characteristic of

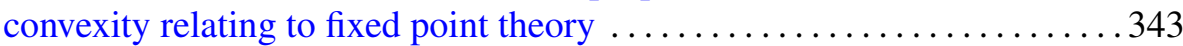

James Arthur Gerhard and Mario Petrich, Word problems for free objects in certain varieties of completely regular semigroups $\ldots \ldots \ldots 351$

Moses Glasner and Mitsuru Nakai, Surjective extension of the reduction

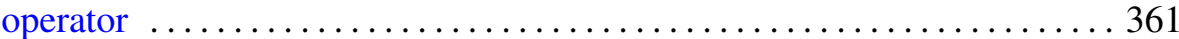

Takesi Isiwata, Ultrafilters and mappings $\ldots \ldots \ldots \ldots \ldots \ldots \ldots \ldots \ldots \ldots \ldots$

Lowell Duane Loveland, Double tangent ball embeddings of curves in $E^{3}$

Douglas C. McMahon and Ta-Sun Wu, Homomorphisms of minimal flows

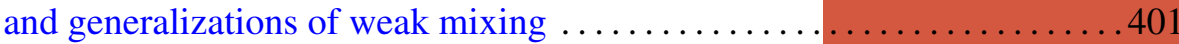

P. H. Maserick, Applications of differentiation of $\mathscr{L}_{p}$-functions to semilattices

Wayne Bruce Powell and Constantine Tsinakis, Free products in the class of abelian $l$-groups

Bruce Reznick, Some inequalities for products of power sums

C. Ray Rosentrater, Compact operators and derivations induced by

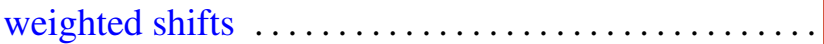

Edward Silverman, Basic calculus of variations

Charles Andrew Swanson, Criteria for oscillatory sublinear Schrödinger equations

David J. Winter, The Jacobson descent theorem 